\title{
Impact of an allied health prehabilitation service for haematologic patients receiving high-dose chemotherapy in a large cancer centre
}

\author{
Jessica Crowe ${ }^{1,2}$ (1) Jill J. Francis ${ }^{3,4,5} \cdot$ Lara Edbrooke $^{1,2} \cdot$ Jenelle Loeliger $^{1} \cdot$ Trish Joyce $^{6} \cdot$ Christina Prickett $^{7}$. \\ Alicia Martin ${ }^{1,3} \cdot$ Amit Khot $^{6,8} \cdot$ Linda Denehy ${ }^{1,2,3}$. the Centre for PrehabilitationPeri-operative Care (CPPOC)
}

Received: 21 April 2021 / Accepted: 29 September 2021 / Published online: 5 October 2021

(c) Crown 2021

\begin{abstract}
Purpose Evaluate the impact of a new multidisciplinary allied health prehabilitation service in haematologic cancer patients receiving high-dose chemotherapy with autologous stem cell transplant (AuSCT).

Methods In a tertiary cancer centre, 12 months of prospectively collected data was retrospectively analysed. Patients were referred to an allied health service for individualised exercise prescription, nutrition intervention and, if indicated through screening, psychological intervention. Impact and operational success were investigated using the RE-AIM framework: patient uptake of the service and sample representativeness (reach); effectiveness in terms of changes in outcomes from initial to pre-transplant assessment; adoption of the service by key stakeholders; fidelity of the prescribed exercise program (implementation); and the extent to which the new service had become routine practice (maintenance).

Results One hundred and eighty-three patients were referred to the AuSCT service over 12 months, of whom 133 (73\%) were referred into the prehabilitation service, 128 (96\%) were eligible and $116(91 \%)$ participated. Patients were representative of Australian AuSCT patients. Eighty-nine patients reached pre-transplant assessment by data censoring; 6-min walk distance $(n=45 / 89,51 \%)$ improved a mean $(95 \%$ CI) of $39.9 \mathrm{~m}$ (18.8 to $61.0, p=<0.005)$ from baseline. Fidelity of exercise prescription was moderate with $72 \%$ of eligible patients receiving the intended exercise interventions. The referral trend over time (maintenance) was high after the initiation period.

Conclusion The prehabilitation service was well adopted by clinicians. Clinically relevant improvements in outcomes were demonstrated. Recommendations, including development of well-integrated discipline-specific assessment intervention and measurement protocols, are highlighted for service improvement. Prehabilitation should be routinely considered to support patients undergoing AuSCT.
\end{abstract}

Keywords Prehabilitation · Implementation · Allied health · Autologous stem cell transplant · High-dose chemotherapy

the Centre for Prehabilitation \& Peri-operative Care (CPPOC) members listed in the supplementary file 1

Jessica Crowe

Jess.crowe@petermac.org

1 Allied Health Department, Peter MacCallum Cancer Centre, Melbourne, VIC, Australia

2 Department of Physiotherapy, The University of Melbourne, Melbourne, VIC, Australia

3 Melbourne School of Health Sciences, The University of Melbourne, Melbourne, VIC, Australia

4 Clinical Epidemiology Program, Ottawa Hospital Research Institute, Ottawa, Canada
5 Department of Health Services Research, Peter MacCallum Cancer Centre, Melbourne, VIC, Australia

6 Department of Clinical Haematology, Peter MacCallum Cancer Centre and Royal Melbourne Hospital, Melbourne, VIC, Australia

7 Department of Clinical Psychology, Psychosocial Oncology Program, Peter MacCallum Cancer Centre, Melbourne, VIC, Australia

8 Sir Peter MacCallum Department of Oncology, The University of Melbourne, Melbourne, VIC, Australia 


\section{Introduction}

The adverse effects of cancer and its treatments are well known and include both physical and psychosocial morbidity [1]. The prevalence and diversity of functional impairments among people with cancer have stimulated the development of intervention strategies that aim to identify and reduce these to maintain or recover pre-diagnosis levels of function [2]. High-dose chemotherapy involves doses associated with myeloablation or significant periods of myelosuppression that require autologous stem cell rescue. This is an intensive treatment for people with haematological cancers, aiming to achieve cure or substantially prolong disease control. Treatment- and disease-related adverse effects for these patients include reduced physical function and health-related quality of life (HRQoL); and increased fatigue, infection, oral mucositis, nausea, and diarrhoea [3].

Cancer prehabilitation is defined as a process on the care continuum prior to major oncological treatment that involves thorough screening, assessment, and treatment of physical and psychological impairments with targeted interventions [4]. Multidisciplinary prehabilitation interventions include optimisation in the control of chronic medical conditions, respiratory education, individualised exercise prescription, nutritional intervention, psychological support and health behaviour change strategies [5]. The aim of cancer prehabilitation is to reduce post-treatment complications, enhance functional capacity and empower the patient to withstand the stressors of their treatment [5]. There is growing evidence in the oncological surgical population supporting prehabilitation [6,7].

Exercise prior to AuSCT is safe and feasible $[8,9]$. Preliminary evidence demonstrates mitigation of the decline in aerobic capacity, muscle strength, HRQoL and increased fatigue that is experienced during conditioning, with the potential for improvements in post-transplant outcomes relating to survival, morbidity and resource utilisation $[9,10]$. Individualised nutrition interventions, coupled with exercise and psychological interventions are likely to be beneficial for this patient cohort [11].

Despite the treatment adverse effects and preliminary efficacy findings, few health services in Australia or indeed world-wide have implemented prehabilitation into routine care. One model that guides assessments of the impact of clinical interventions is 'RE-AIM' (reach, effectiveness, adoption, implementation, maintenance). This international framework was developed over 20 years ago [12] and is now often used to plan and assess the translation of interventions into practice in clinical settings $[13,14]$.

At the participating centre, the anaesthetics department established a prehabilitation service approximately
10 years ago for high-risk surgical patients. The current study examined the impact, over 12 months, of adding a standardised multidisciplinary allied health prehabilitation clinical service as standard care for haematologic cancer patients receiving intensive chemotherapy prior to AuSCT.

\section{Methods}

\section{Setting and study design}

A retrospective evaluation of a tertiary cancer centre cohort analysing prospective data collected between March 2019 (allied health service commencement) to March 2020. The model of standard care is detailed in Fig. 1.

\section{Program development}

Staff delivering the service were physiotherapists/exercise physiologists (EPs), dietitians and clinical psychologists with an average of 3,5 and 4 years specialist oncology experience respectively. Further information about program development and staff training is in supplementary file 2 .

\section{Procedure}

Intervention component reporting follows the Template for Intervention Description and Replication (TIDiER) [15] guidelines as described in supplementary file 2 .

\section{Patients, referral and enrolment}

Eligible patients were adults being considered for high-dose chemotherapy (majority receiving high-dose melphalan or Stanford BCNU/carmustine regimen) and AuSCT in the haematology service who had nil contraindications to exercise and were cognitively able to complete an independent home exercise program.

The planned referral pathway involved the AuSCT transplant haematologists and nurse consultants referring all eligible patients following acceptance into the AuSCT service by haematologists. Patients were telephone screened by a clinician, for exercise program eligibility using the validated Australian-modified Karnofsky Performance Status (AKPS) [16]. Scores range between 10 and 100 (higher reflecting improved function), based on the patients' ability to perform regular tasks relating to activity, work, and self-care. Patients with an AKPS $<50$ were ineligible. Patients with bone involvement were eligible if cleared to exercise by their haematologist. Psychology eligibility screening was completed using the patient health questionnaire (PHQ-4), a valid tool for detecting anxiety and depressive disorders 


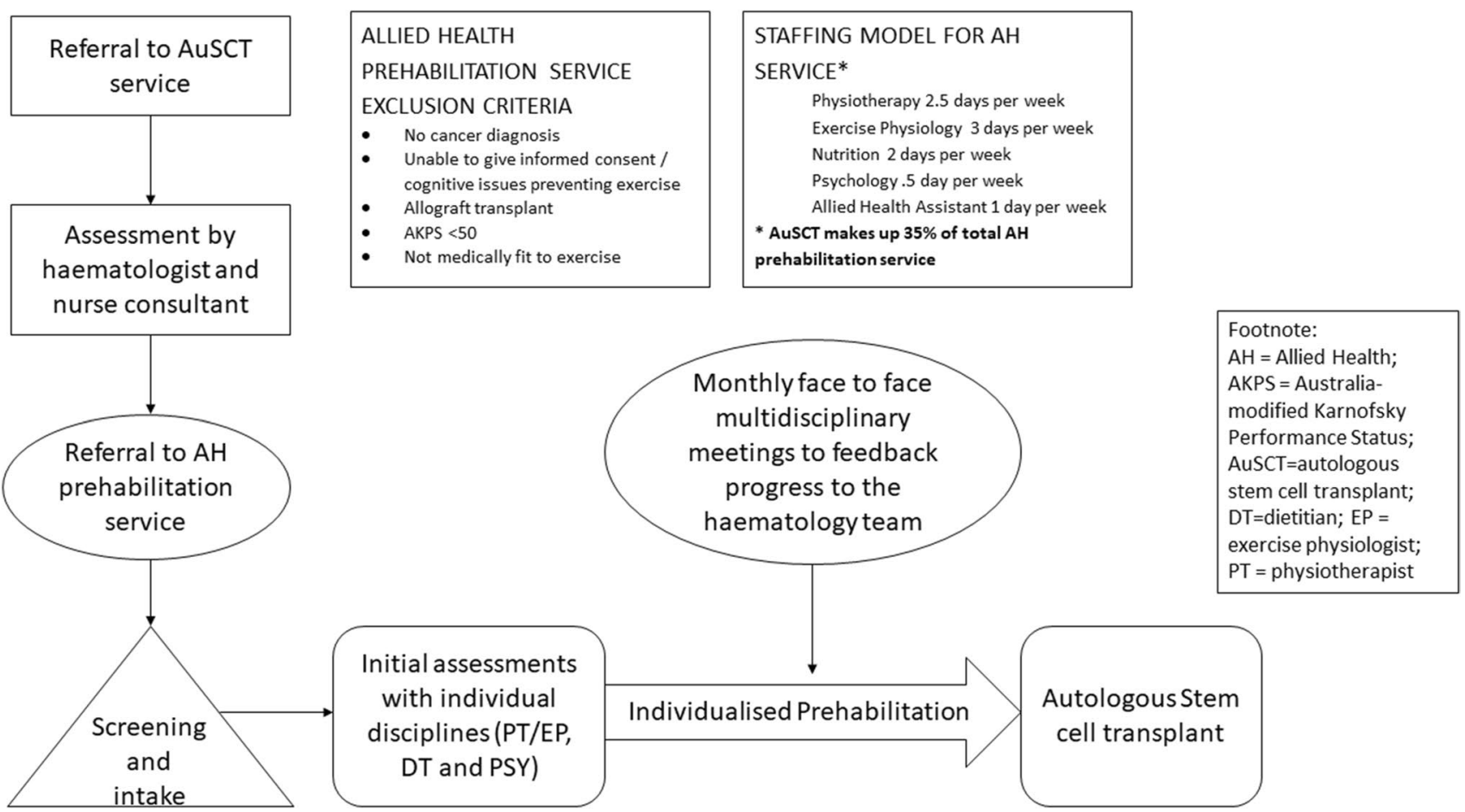

Fig. 1 Allied health prehabilitation model of care for AuSCT patients

[17]. Patients were eligible for clinical psychology if they scored $>2$ (mild distress).

Eligible patients were invited to participate and those accepting were booked for individual assessments with each discipline and clinical psychologist if eligible. Program duration was individualised, depending on the timing of referral in relation to medical treatment interventions.

\section{Exercise intervention}

An initial, individual assessment (60 min) at the hospital was conducted. Supplementary file 2 presents safety criteria for starting and ceasing supervised exercise assessments/exercise. Treatment was commenced at initial assessment after baseline measures were completed. An individually tailored exercise program consisting of continuous moderate intensity (aiming for a moderate-high intensity '4-5-somewhat hard' on the modified Borg Scale) aerobic and resistance exercises was prescribed. The exercise intervention was based upon initial assessment findings and aimed to progress towards meeting the Exercise and Sports Science Australia [18] recommendations of 150 min of moderate intensity aerobic exercise and two resistance exercise sessions per week. Supplementary file 2 provides further details. Patients completed the program either independently at home, in a local gym, supervised at a community health centre or at the hospital gym. The program location was determined by patient residential location and preference and level of supervision needed. Programs were progressed to maintain moderate intensity, with weekly/fortnightly telehealth or phone reviews. Program adherence was monitored using patient self-report. Patients did not receive intervention from a physiotherapist during AuSCT admission unless referred.

\section{Nutrition intervention}

Initial assessment considered relevant clinical and anthropometric markers, dietary intake and estimation of nutritional requirements. This included height, weight and body mass index which allowed tracking of percentage weight change. The nutrition intervention consisted of 20-min individualised sessions (hospital or phone), with the goals of care including maintenance/improvement in nutrition status. Intervention included medical nutrition therapy, information about the benefits of nutrition prehabilitation, prescription of relevant therapeutic diet, educational materials (supplementary file 2) and nutrition prehabilitation goal setting. Between 1 and 4 follow-up appointments were scheduled if indicated at initial assessment, and treatment modifications were based on outcome changes. On hospitalisation for AuSCT, patients received standard care which included automatic referral to dietitians for nutritional monitoring and intervention as required, 


\section{Clinical psychology intervention}

A full psychological initial assessment (60 min, hospital) with individualised psychological support based on findings. Between 1 and 7 hospital follow-up appointments were scheduled and interventions included cognitive behavioural approaches to managing worry and pain, maintaining motivation and activating supports in preparation for AuSCT. Patients did not receive psychology intervention during hospitalisation for AuSCT.

\section{Outcomes}

Evaluation of the impact of the prehabilitation program was measured using the RE-AIM framework (reach, effectiveness, adoption, implementation and maintenance). Table 1 describes the evaluation process for each dimension of the framework. Effectiveness outcomes are described below.

\section{Effectiveness outcomes as part of the RE-AIM framework measurements}

Patients completed outcome measures at baseline (T0, initial assessment with prehabilitation team), pre-transplant (T1, assessment window within 14 days of AuSCT but planned around other medical appointments) and 30 days post-AuSCT (T2, day 0 being re-infusion of patients cells). The 6MWT is a commonly used submaximal test of functional exercise capacity [22] and is valid and reliable in cancer patients [23]. The 6MWT was completed using a $30-\mathrm{m}$ corridor and standardised encouragement. The Baseline Lite Hydraulic handgrip dynamometer (Fabrication Enterprises Inc.) was used to assess handgrip strength and data from this device correlates with the Jamar dynamometer [24], which has demonstrated validity and reliability in the oncology population [25]. The validated 30-s sit-tostand test (30secSTS), performed according to standard protocols, assessed lower limb strength and function [26].

Nutritional status was measured using the valid and reliable Patient-Generated Subjective Global Assessment (PG-SGA) [27] and percentage weight change. HRQoL was measured using the European Organisation for the Research and Treatment of Cancer Quality of Life Questionnaire Core 30 (EORTC-QLQ-C30) [28]. The International Physical Activity Questionnaire-Short Form (IPAQ-SF) is a reliable and valid [29] self-reported tool that assesses physical activity (PA) level.

Table 1 Outcome measurement based on the RE-AIM framework [13]

\begin{tabular}{|c|c|}
\hline Dimension & Explanation \\
\hline Reach & $\begin{array}{l}\text { Includes the proportion of eligible patients who participate in the program being evaluated, and their representativeness rela- } \\
\text { tive to a wider group of eligible patients. We compared the number and characteristics of the patients who were referred } \\
\text { to the AuSCT team but did not go onto referral to allied health prehabilitation services, with those who participated in the } \\
\text { service and those who declined the service. This included patient location, categorised into metropolitan, regional and remote } \\
\text { [19]. These characteristics were then compared to data of AuSCT patients Australia wide }\end{array}$ \\
\hline Effectiveness & $\begin{array}{l}\text { Objective and patient-reported outcomes from initial assessment to pre-transplant assessment and 30-days after transplant were } \\
\text { recorded. Tests were standardised and treating clinicians received verbal and written education on each outcome individually } \\
\text { prior to service commencement. Further details regarding outcomes are provided in text }\end{array}$ \\
\hline Adoption & The number of referrals received was expressed as a percentage of the overall number of eligible patients at the hospital \\
\hline Implementation & $\begin{array}{l}\text { Despite the well reported importance of intervention fidelity to allow accurate interpretation of results, the quality of interven- } \\
\text { tion fidelity reporting remains poor, especially in exercise interventions [15]. With this in mind, assessment of implementa- } \\
\text { tion (fidelity) involved a retrospective audit of the } 116 \text { patients who participated in the exercise prehabilitation program to } \\
\text { review how many patients received the exercise intervention as described in the protocol } \\
\text { We accessed the patients' medical records to review the initial assessment with an exercise professional; record of modality of } \\
\text { exercise prescribed as well as the exercise parameters that were documented, based upon the frequency, intensity, time and } \\
\text { type (FITT) exercise principles, was assessed. If there was a documented reason why the intended program was not initially } \\
\text { prescribed the next documented exercise appointment was used to evaluate fidelity. Based on consensus in the literature we } \\
\text { regarded 'high fidelity' as } 80 \text { to } 100 \% \text { of patients being prescribed an aerobic and resistance individualised home exercise } \\
\text { program, with 51-79\% representing moderate fidelity of delivery and } \leq 50 \% \text { deemed low fidelity of delivery [20] } \\
\text { For further measures of fidelity, we recorded the mean number of sessions attended for each discipline compared to the number } \\
\text { of scheduled sessions (attendance rate) for each discipline, and the percentage of people attending supervised exercise class } \\
\text { compared to home-based exercise including the mean number of supervised classes attended }\end{array}$ \\
\hline Maintenance & $\begin{array}{l}\text { We assessed the extent to which referral to the new allied health services within the prehabilitation service had become routine } \\
\text { practice. We define 'routine' as clinical actions that have been performed for } 12 \text { months [21] } \\
\text { This involved a descriptive analysis of the trend in referrals over time compared to the number of eligible patients. Patients } \\
\text { referred to the AuSCT team from January } 2019 \text { onwards were eligible for referral into the prehabilitation service if they were } \\
\text { scheduled to receive their transplant at least } 3 \text { weeks post service commencement }\end{array}$ \\
\hline
\end{tabular}




\section{Data management and analysis}

A research electronic data capture (REDCap) database was used [30]. Descriptive statistics were reported as counts and percentages, means and standard deviations or medians and interquartile ranges, as appropriate, to summarise variables. The change in objective and patient-reported outcomes from initial assessment (baseline) to pre-procedure was assessed using paired $t$-tests or the Wilcoxon signed rank test depending on the data distribution normality (continuous) and $\mathrm{Mc}$ Nemar's test (categorical). No analyses involved all three time points. All tests of significance $(p<0.05)$ performed are described and no adjustment was made for the number of outcomes assessed [31].

\section{Results}

Findings relating to each of the dimensions of the RE-AIM framework are described in turn below.

\section{Reach}

In 2018, 1180 adult ( $>15$ year olds) patients underwent an AuSCT in Australia, 25\% (296) of these were in the state of Victoria [32]. The study hospital performs an average of 140 AuSCT a year, approximately half of all Victorian transplants. Demographic and clinical characteristics of Australian AuSCT recipients included 55\% were $\geq 60$ years of age, $61 \%$ male* $(*$ this gender percentage includes allograft transplant patients) and were diagnosed with multiple myeloma (57\%) or lymphoma (35\%) [32]. Patients who took up the allied health prehabilitation service were broadly representative of Australian data with $65 \%$ being $\geq 60$ years of age, $64 \%$ male, and diagnosed with multiple myeloma (64\%) or lymphoma (32\%).

Figure 2 gives the flow of participants through the study, $73 \%$ of patients referred to the AuSCT service were referred onto prehabilitation. Of the 133 patients referred to the service, five were ineligible, and another seven declined.

Baseline characteristics of patients who participated, those who declined the service, and patients who were referred to the AuSCT service but not referred to the allied health prehabilitation service between March 2019 and March 2020 are given in Table 2. Participating patients were a median age of 64 years, predominantly had multiple myeloma and one-third were rurally located. When comparing demographic (age, sex) and clinical (diagnosis) characteristics at a hospital level, the only significant difference was the age of patients referred compared to those who were not
Fig. 2 Referral, uptake and receipt of intervention to assess reach

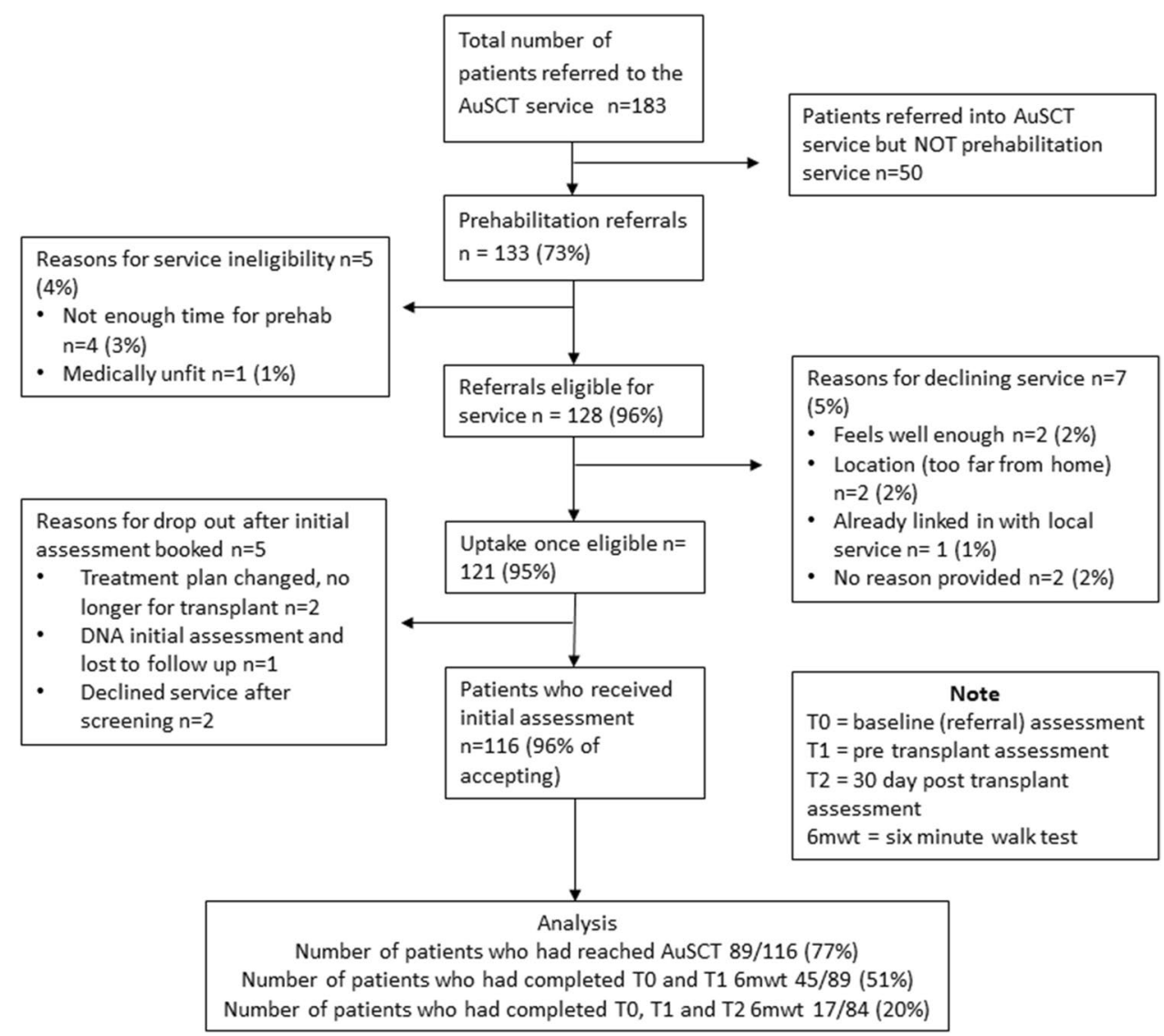


Table 2 Baseline characteristics of patients

\begin{tabular}{|c|c|c|c|}
\hline & \multicolumn{2}{|l|}{ Referred patients } & \multirow[t]{2}{*}{ Non-referred patients } \\
\hline & Patients accepting referral & Patients declining referral & \\
\hline $\mathrm{n}$ & 121 & 7 & 50 \\
\hline Age at referral, years median (IQR) & $64.0(56.0-69.0)$ & $62.0(54.0-64.0)$ & $58.0(52.8-65.3)$ \\
\hline Sex $n(\%)$ male & $64(52.9 \%)$ & $5(71.4 \%)$ & $32(64 \%)$ \\
\hline Cancer diagnosis $n(\%)$ & $\begin{array}{l}\text { Multiple myeloma } \\
78(64.5 \%) \\
\text { Lymphoma } 39(32.2 \%) * \text { Other } 4 \\
\quad(3.3 \%)\end{array}$ & $\begin{array}{l}\text { Multiple myeloma } \\
3(42.9 \%) \\
\text { Lymphoma } 4(57.1 \%)\end{array}$ & $\begin{array}{l}\text { Multiple myeloma } \\
25(50.0 \%) \\
\text { Lymphoma } 24 \\
\quad(48.0 \%) * \text { Other } 1 \\
(2.0 \%)\end{array}$ \\
\hline $\begin{array}{l}\text { Received neoadjuvant treatment during } \\
\text { prehabilitation, } n(\%) \text { 'yes' }\end{array}$ & $86 / 115(74.8 \%)$ & & \\
\hline Location $n(\%) \#$ & $\begin{array}{l}\text { Metro: } 79(65.3 \%) \\
\text { Rural: } 41(33.9 \%) \\
\text { Remote: } 1(1.0 \%)\end{array}$ & $\begin{array}{l}\text { Metro: } 4(57.1 \%) \\
\text { Rural: } 3(42.9 \%) \\
\text { Remote: } 0(0.0 \%)\end{array}$ & $\begin{array}{l}\text { Metro: } 21(42.0 \%) \\
\text { Rural: } 27(54.0 \%) \\
\text { Remote: } 2(4.0 \%)\end{array}$ \\
\hline
\end{tabular}

referred (age (years), median (IQR): referred $(n=131) 63$ $(56,69)$ versus not referred $58(53,65), p=0.011)$. There were no significant differences with respect to age, sex or diagnosis for those who took up referral compared to those who declined referral. Location of patients as metro, rural or remote is given in Table 2. There was a higher percentage of rural and remote patients in the non-referred patient cohort $(29 / 50,58 \%)$ compared with the referred patients $(47 / 133$, $35 \%), p=0.009$.

\section{Effectiveness}

Table 3 presents results of objective measures and patientreported outcomes for patients who completed assessments at baseline, pre-procedure and 30-day post-procedure timepoints. Baseline data collection for this evaluation was censored on March 12, 2020. Eighty-nine of the 116 (77\%) patients participating in prehabilitation had undergone their AuSCT, and $84(72 \%)$ were $>30$ days post-AuSCT at data analysis. No serious adverse events occurred. The number of patients who completed outcome measures at T0 and T1 was low; 45/89 (51\%) completed the 6MWT at both timepoints (Table 3). The time between initial and pre-transplant assessment 6MWT performance was a median (interquartile range) of 48.5 (33.0 to 91.0) days. For patients who did complete assessments at both T0 and T1 functional exercise capacity, lower limb strength and function, handgrip strength and physical activity levels improved significantly following prehabilitation (Table 3). The 6MWT distance and 30secSTS changes were a mean difference $(95 \% \mathrm{CI})$ of $39.9 \mathrm{~m}(18.8$ to $61.0, p=<0.005)$ and median (IQR) of 13.0 stands
(10.5-16.0) versus 15.0 stands (13.0-18.0), $p=<0.005$, respectively. Significantly more patients reported meeting the physical activity guidelines following prehabilitation (T0 28\% versus T1 52\%, $p=0.002$ ). Global HRQoL was not significantly different following prehabilitation (significant improvements in the fatigue symptom scale $(p=0.005)$ and dyspnoea single item $(p=0.018)$ ) (Table 3$)$.

Thirty-day outcome data were not evaluated statistically due to attrition at this timepoint; 17/84 (20\%) patients had completed the 6MWT at the three timepoints.

\section{Adoption}

As reported in Fig. 2, 73\% $(n=133)$ were referred to the allied health prehabilitation service. Of the total numbers referred, 92\% $(n=122)$ were received from the haematology (including AuSCT) nurse consultants as per the planned referral pathway. The remaining referrals were received from haematology consultants $(7.5 \%, n=10)$ and an allied health professional $(0.5 \%, n=1)$.

\section{Implementation}

As discussed in Table 1, exercise prescription was the focus in the implementation domain. Of the 116 patients who received an initial assessment with an exercise clinician; $72 \%(n=84)$ were prescribed a combined aerobic and resistance home-based exercise program. This represents moderate fidelity of delivery [20]. However, reporting of the exercise parameters for each domain varied between 0 and $85 \%$ (supplementary file 3 ), making it difficult to analyse 


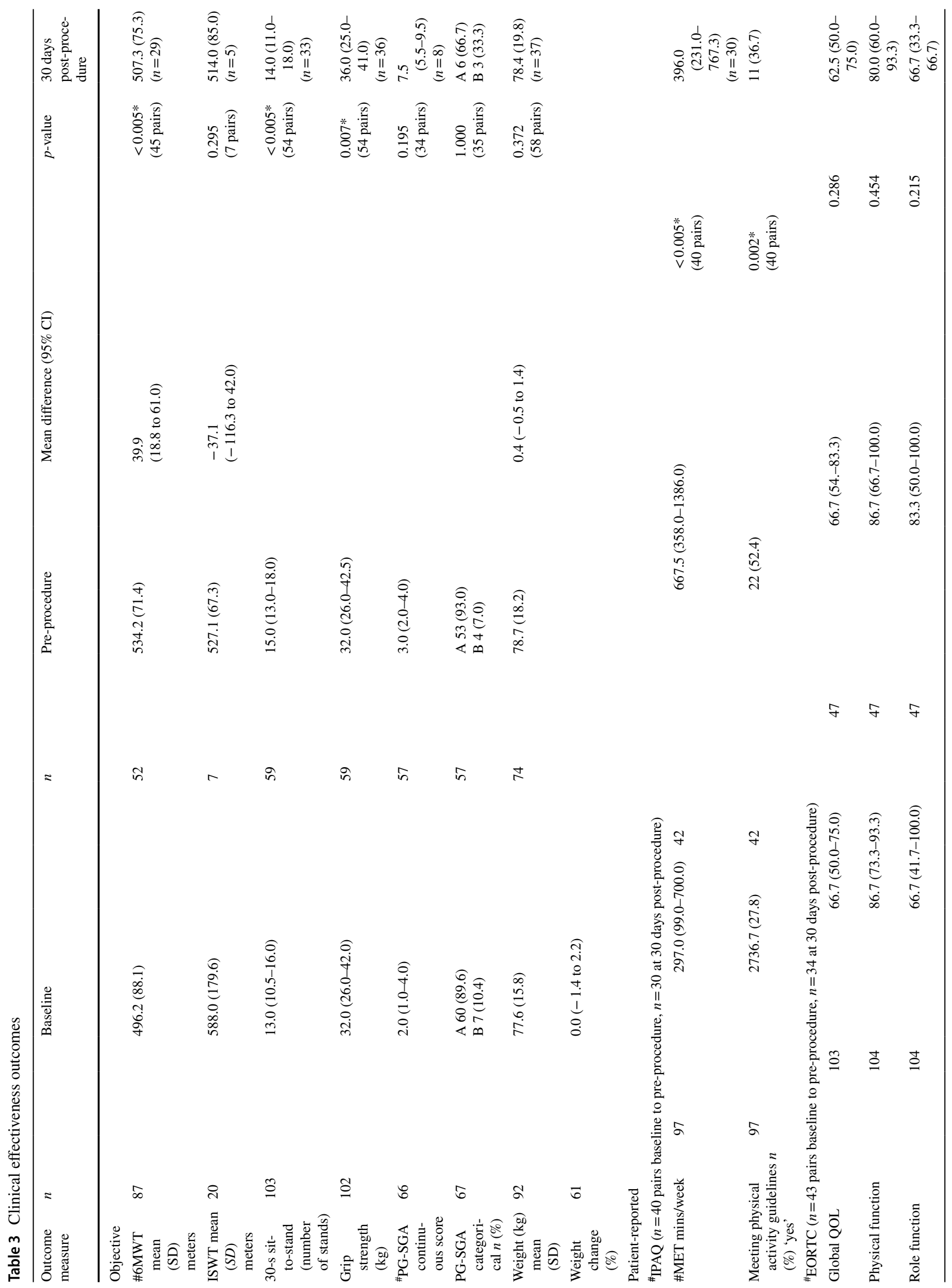




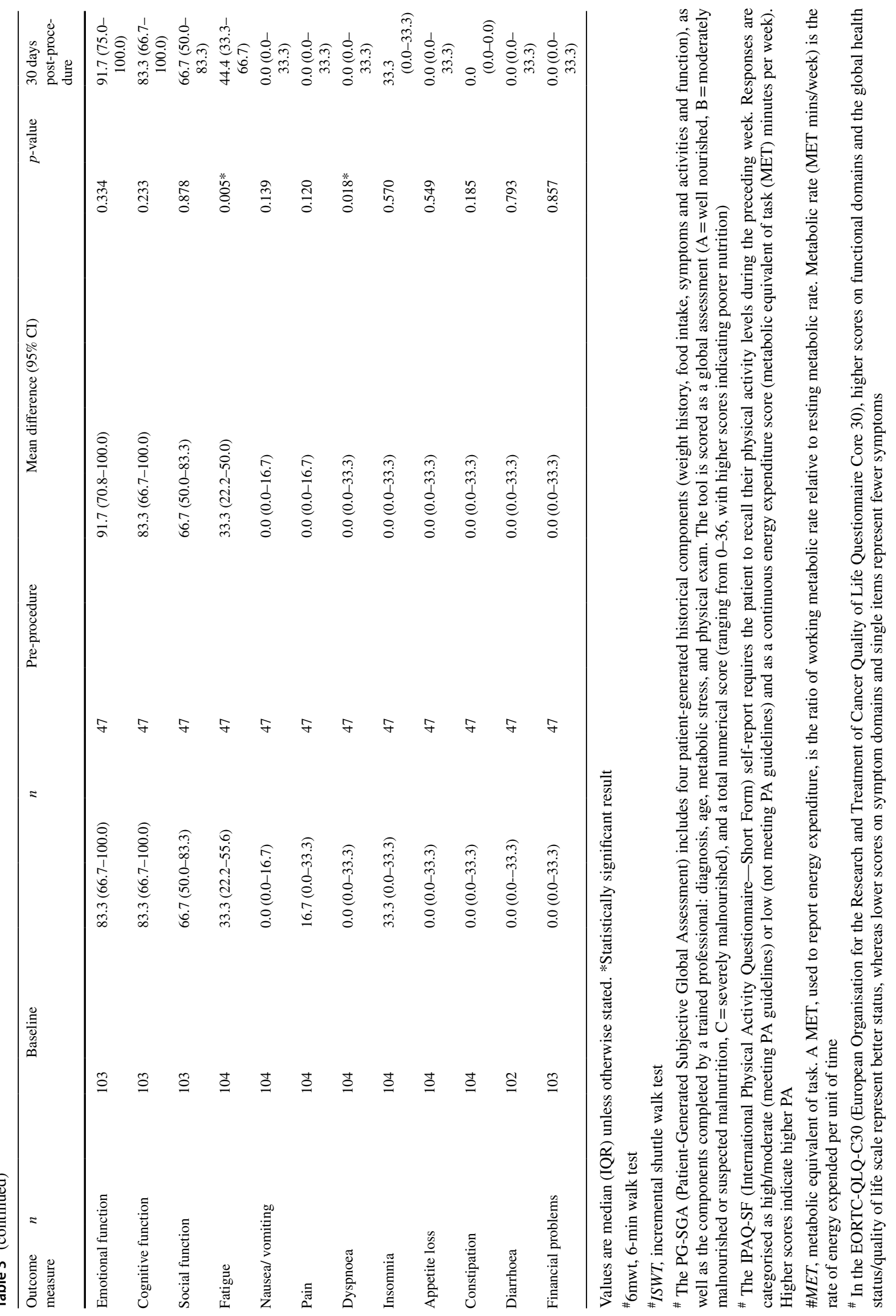


'true' fidelity of delivery. We reviewed 15 records with the lowest fidelity (those where aerobic and/or resistance exercise was prescribed but no parameters were documented in the medical records) and compared medical record documentation to documentation recorded separately using the physiotherapy exercise prescription software, SimpleSet [34]. We found exercise prescription parameters were more consistently reported in SimpleSet compared to the medical records especially in the resistance programs where $62.5 \%$ of records had all parameters documented on SimpleSet when not documented in the medical records.

The mean attendance rates for individual disciplines appointments was high ranging from 80 to $92 \%$ (supplementary file 4$)$. Eighteen percent $(n=21)$ of patients attended supervised group exercise class at the hospital, and on average attended 5.7 group exercise sessions during their prehabilitation period.

\section{Maintenance}

The number of patients referred to the prehabilitation service over the 12 months compared to total referrals into the AuSCT team was $73 \%$ (monthly breakdown in supplementary file 5); however, it can be broken down further into three key time periods:

1. Service initiation including communication and promotion of the service to the relevant stakeholders and referrers (Jan-April 2019) - referral rate 52\%.

2. Service maintenance (May 2019-Jan 2020)—referral rate $81 \%$

3. COVID-19 impact (Feb-March 2020)-referral rate $56 \%$.

\section{Discussion}

In this study, the RE-AIM framework [13] was applied to evaluate the impact and operational success of the service. Findings relating to all five dimensions of the framework inform the judgement as to the current impact of this service and avenues for enhancement of future, improved services for this population. In summary, the sample was representative of Australian AuSCT patients, and effectiveness was demonstrated with improvements at follow-up for the 6MWT, 30secSTS, grip strength and several patientreported outcomes. However, modifications are required to increase outcome measure completion as there was a high level of missing data. Adoption of the service by referrers, at $73 \%$, was moderate to high. Implementation (fidelity of exercise intervention delivery) was moderate; however, this assessment relied on documentation in patients' case notes, which may have been suboptimal; not all components of exercise prescription principles (frequency, intensity, time, type) were routinely documented. The referral trend over time (maintenance) was high after the initiation period, although interrupted by the onset of COVID-19 restrictions in March 2020. Based on the findings from the RE-AIM analysis, the impact of the prehabilitation intervention in this patient group was high, with reason to infer that impact on the wider population of eligible patients across the nation would be similarly moderate to high, if sites were to initiate this service. The framework provided a feasible, comprehensive, and informative approach to evaluating impact.

There is emerging evidence to suggest that multimodal prehabilitation should be initiated as an oncology health service [35]. More than $90 \%$ of eligible, referred patients went on to participate in the allied health prehabilitation service, demonstrating excellent uptake. Our results are consistent with those from the UK where uptake of cancer prehabilitation prior to gastrointestinal and lung surgery was high [35]. This finding could be attributed to an engaged haematology service, this hypothesis is strengthened by the extent of adoption from these referrers. Prior to the COVID-19 pandemic, the referral rate into the service over the previous nine months was $>80 \%$ of eligible patients. This excellent adoption contrasts with current evidence regarding oncology clinicians' referral patterns to exercise specialists [36].

The current study, conducted in real-world conditions, reports measurable improvements with allied health prehabilitation in functional exercise capacity, lower limb strength and function, grip strength, physical activity and HRQoL domains (fatigue and dyspnoea). This is consistent with two small studies in allogenic and AuSCT, where positive trends were found for prehabilitation in physical activity, physical function, HRQoL and fatigue $[9,10]$ and a significant positive change in lean body weight [10]. Our findings should be interpreted with caution due to the absence of a control group. Furthermore, there is no reported minimal clinical important difference (MCID) for the outcome measures used specific to haematology oncology patients. Our 6MWT difference of $40 \mathrm{~m}$ is consistent with clinically significant differences in similar populations (22-42 m, lung cancer) [37]. However, given the small patient numbers and the fact that only one 6MWT was performed at each assessment, interpretation of the results should be interpreted with caution, as there is a known learning effect with the 6MWT [38].

It was difficult to evaluate the nutrition intervention due to missing data. However, it is clinically appropriate for a patient's nutritional status to be maintained or improved whilst receiving prehabilitation nutrition therapy and this was observed in this patient cohort [11].

There are many well-documented challenges around translating research findings into routine practice in health care [39]. For effective and sustained system change in health care, sophisticated implementation strategies must 
be paired with timely and relevant data collection [39]. A notable challenge during the evaluation of this service was effective and timely patient-level data collection. Only $51 \%(45 / 89)$ of patients completed the 6MWT at baseline and pre-procedure. Indeed, the final time point at 30 days after AuSCT had significant attrition (17/84 (20\%) 6MWT completion). This could lead to bias as it is possible that a more engaged cohort of patients returned for their followup assessments. This is likely to be due to multiple factors including lack of clinician time, administrative resources, the COVID-19 pandemic restricting hospital appointments, and patient factors of residential distance from the centre (35\% of the patients were classified as either rural or remote), disease status, treatment toxicity and appointment burden [40]. Considering this, dedicated administrative funding should be built into any budget bid for development of future multidisciplinary prehabilitation programs.

An important consideration when assessing the credibility of an effectiveness study is intervention fidelity [41]. High intervention fidelity helps prevent incorrect or inaccurate interpretation of the results [42]. Despite the importance of this, the quality of intervention fidelity reporting generally remains poor [15]. We used the implementation dimension of the RE-AIM framework to evaluate this and similar to Poltawski et al. 2014 [41] found assessing intervention fidelity difficult. Only $72 \%$ of eligible patients received the combined aerobic and resistance exercise intervention. Documentation of exercise parameters was variable ( $0-85 \%)$ and could be due to several factors including time constraints, exercise templates which did not require all exercise parameters be recorded, and staff priorities. Based on the SimpleSet [34] documentation, we can extrapolate that the documentation of exercise prescription parameters in the medical records may underestimate the 'true' level of fidelity, as apparent low fidelity may be attributable to reporting omissions. With the initiation of electronic medical records (EMR) in August 2020, data is now directly fed from SimpleSet into the EMR. A further limitation of the study was our uni-dimensional assessment of implementation fidelity, evaluating clinicians' delivery of the intervention, without having the tools to review patients' adherence to the prescribed program.

Waterland et al. 2020 [43] found $72 \%$ of patients prior to oncological surgery would prefer exercise prehabilitation in a home-based environment. Although initial work has been done to optimise the home-based service, especially in the wake of the COVID-19 pandemic, further scoping needs to occur involving app-based, web-based or virtual reality methodologies to improve treatment fidelity when exercising at home [44].

Eight recommendations for developing a multidisciplinary prehabilitation program are provided in Box 1. The next steps are to evaluate the fidelity of the nutrition and psychology interventions and to examine the hard endpoints for benefit of the program, including morbidity, infections and mortality indicators, length of hospital stay and patient satisfaction. A powered multidisciplinary randomised controlled study would raise the level of evidence relating to interventions in this patient population.

Box 1. Eight recommendations for developing a multidisciplinary prehabilitation program

Prior to service implementation:

$\bigcirc$ Ensure adequate administrative staffing to allow for timely scheduling of appointments and to minimise patients lost to follow-up

Provide all relevant allied health staff with the training and resources required to conduct face to face and telehealth assessments and interventions

In addition to offering supervised exercise classes, consider virtual exercise classes via telehealth to service rural and remote patients

Ensure adequate implementation and communication strategies are utilised for service promotion to key referrers

For exercise prehabilitation:

OWhere possible revise medical record templates to improve exercise prescription documentation, especially pertaining to FITT principles. Train relevant staff regarding the importance of this Following implementation: OReview patient prehabilitation outcomes as part of the clinical decision-making process regarding fitness for transplant OReview processes every two months and update clinical training to ensure consistency with prescription and outcome measurement OConduct an annual formal evaluation of the program with all stakeholders with a view to assessment of impact and continued service improvement

\section{Conclusion}

The allied health prehabilitation service was well adopted by patients and clinicians and sustainable within the haematology and allied health services. It demonstrated clinically relevant improvements in outcomes, although gaps in data collection and intervention fidelity need to be addressed. The service continues to evolve based on these findings, on-going patient and clinician feedback, and as the body of research evidence increases. This model and recommendations can be used to inform the development of future multidisciplinary prehabilitation clinics in haematology and other cancer patient populations.

Supplementary Information The online version contains supplementary material available at https://doi.org/10.1007/s00520-021-06607-w.

Acknowledgements We would like to thank all the Peter MacCallum multidisciplinary teams involved in the development, implementation, and evaluation of the AuSCT prehabilitation service and the CPPOC team for the facilitation and continuation of excellence in prehabilitation care.

Author contributions AM developed the successful allied health business case that led to the implementation of the service. AK, TJ, 
LD, LE, JC, JL, CP and AM contributed to the service development, implementation and evaluation. JC and LE contributed to the data collection and storage. LD, JF, LE, AK and JC contributed to the data analysis, interpretation and the design of the study. JC completed this manuscript as part of her postgraduate research through the University of Melbourne. JC is supervised by LD, JF, LE and AK. All authors contributed to the manuscript writing and had final approval of the manuscript. The CPPOC group represents the multidisciplinary team of clinicians at Peter MacCallum Cancer Centre that either contributed to the development of the prehabilitation service (surgical and haematology cohorts) or who are clinically responsible for the daily operations of the service and patient care. For further details about CPPOC please contact sonia.coleman@ petermac.org.

Data availability The datasets used and/or analysed during the current study are available from the corresponding author on reasonable request.

\section{Declarations}

Ethics approval and consent to participate The study received ethical approval by the Peter MacCallum Cancer Centre human research ethics committee on 6th October 2020, Project ID: QA/67920/PMCC-2020. Patient and implementation data were collected during usual clinical care, so no patient consent was required, and the study conducted in accordance with the Declaration of Helsenski.

Conflict of interest The authors declare no competing interests.

\section{References}

1. Soerjomataram I, Lortet-Tieulent J, Parkin DM, Ferlay J, Mathers C, Forman D et al (2012) Global burden of cancer in 2008: a systematic analysis of disability-adjusted life-years in 12 world regions. Lancet 380(9856):1840-1850

2. Carli F, Zavorsky GS (2005) Optimizing functional exercise capacity in the elderly surgical population. Curr Opin Clin Nutr Metab Care 8(1):23-32

3. Chakraborty R, Hamilton BK, Hashmi SK, Kumar SK, Majhail NS (2018) Health-related quality of life after autologous stem cell transplantation for multiple myeloma. Biol Blood Marrow Transplant 24(8):1546-1553

4. Silver JK, Baima J (2013) Cancer prehabilitation: an opportunity to decrease treatment-related morbidity, increase cancer treatment options, and improve physical and psychological health outcomes. Am J Phys Med Rehabil 92(8):715-727

5. Macmillan Cancer Support. Prehabilitation for people with cancer 2017

6. Gillis C, Li C, Lee L, Awasthi R, Augustin B, Gamsa A et al (2014) Prehabilitation versus rehabilitation: a randomized control trial in patients undergoing colorectal resection for cancer. Anesthesiology 121(5):937-947

7. West MA, Loughney L, Lythgoe D, Barben CP, Sripadam R, Kemp GJ et al (2015) Effect of prehabilitation on objectively measured physical fitness after neoadjuvant treatment in preoperative rectal cancer patients: a blinded interventional pilot study. Br J Anaesth 114(2):244-251

8. Wood WA, Phillips B, Smith-Ryan AE, Wilson D, Deal AM, Bailey $C$ et al (2016) Personalized home-based interval exercise training may improve cardiorespiratory fitness in cancer patients preparing to undergo hematopoietic cell transplantation. Bone Marrow Transplant 51(7):967-972
9. van Haren I, Staal JB, Potting CM, Atsma F, Hoogeboom TJ, Blijlevens NMA et al (2018) Physical exercise prior to hematopoietic stem cell transplantation: a feasibility study. Physiother Theory Pract 34(10):747-756

10. Coleman EA, Coon S, Hall-Barrow J, Richards K, Gaylor D, Stewart B (2003) Feasibility of exercise during treatment for multiple myeloma. Cancer Nurs 26(5):410-419

11. Arends J, Bachmann P, Baracos V, Barthelemy N, Bertz H, Bozzetti $F$ et al (2017) ESPEN guidelines on nutrition in cancer patients. Clin Nutr 36(1):11-48

12. Glasgow RE, Vogt TM, Boles SM (1999) Evaluating the public health impact of health promotion interventions: the RE-AIM framework. Am J Public Health 89(9):1322-1327

13. Glasgow RE, Harden SM, Gaglio B, Rabin B, Smith ML, Porter GC et al (2019) RE-AIM Planning and evaluation framework: adapting to new science and practice with a 20 -Year review. Front Public Health 7:64

14. Gaglio B, Shoup JA, Glasgow RE (2013) The RE-AIM framework: a systematic review of use over time. Am J Public Health 103(6):e38-46

15. Hoffmann TC, Glasziou PP, Boutron I, Milne R, Perera R, Moher $D$ et al (2014) Better reporting of interventions: template for intervention description and replication (TIDieR) checklist and guide. BMJ 348:g1687

16. Abernethy AP, Shelby-James T, Fazekas BS, Woods D, Currow DC (2005) The Australia-modified Karnofsky Performance Status (AKPS) scale: a revised scale for contemporary palliative care clinical practice [ISRCTN81117481]. BMC Palliat Care 4:7

17. Kroenke K, Spitzer RL, Williams JB, Lowe B (2009) An ultrabrief screening scale for anxiety and depression: the PHQ-4. Psychosomatics 50(6):613-621

18. Hayes SC, Newton RU, Spence RR, Galvao DA (2019) The exercise and sports science Australia position statement: exercise medicine in cancer management. J Sci Med Sport 22(11):1175-1199

19. Department of Primary Industries and Energy and Department of Human Services and Health (1994) Rural, remote and metropolitan areas classification (RRMA). Commonwealth of Australia, Canberra

20. Borrelli B (2011) The assessment, monitoring, and enhancement of treatment fidelity in public health clinical trials. J Public Health Dent 71(Suppl 1):S52-63

21. Brewster AL, Curry LA, Cherlin EJ, Talbert-Slagle K, Horwitz LI, Bradley EH (2015) Integrating new practices: a qualitative study of how hospital innovations become routine. Implement Sci 10:168

22. Holland AE, Spruit MA, Troosters T, Puhan MA, Pepin V, Saey D et al (2014) An official European Respiratory Society/American Thoracic Society technical standard: field walking tests in chronic respiratory disease. Eur Respir J 44(6):1428-1446

23. Schmidt K, Vogt L, Thiel C, Jager E, Banzer W (2013) Validity of the six-minute walk test in cancer patients. Int J Sports Med 34(7):631-636

24. Mathiowetz V, Kashman N, Volland G, Weber K, Dowe M, Rogers S (1985) Grip and pinch strength: normative data for adults. Arch Phys Med Rehabil 66(2):69-74

25. Trutschnigg B, Kilgour RD, Reinglas J, Rosenthall L, Hornby L, Morais JA et al (2008) Precision and reliability of strength (Jamar vs. Biodex handgrip) and body composition (dual-energy $\mathrm{X}$-ray absorptiometry vs. bioimpedance analysis) measurements in advanced cancer patients. Appl Physiol Nutr Metab. 33(6):1232-9

26. Jones CJ, Rikli RE, Beam WC (1999) A 30-s chair-stand test as a measure of lower body strength in community-residing older adults. Res Q Exerc Sport 70(2):113-119

27. Bauer J, Capra S, Ferguson M (2002) Use of the scored PatientGenerated Subjective Global Assessment (PG-SGA) as a 
nutrition assessment tool in patients with cancer. Eur J Clin Nutr 56(8):779-785

28. Aaronson NK, Ahmedzai S, Bergman B, Bullinger M, Cull A, Duez NJ et al (1993) The European Organization for Research and Treatment of Cancer QLQ-C30: a quality-of-life instrument for use in international clinical trials in oncology. J Natl Cancer Inst 85(5):365-376

29. Craig CL, Marshall AL, Sjostrom M, Bauman AE, Booth ML, Ainsworth BE et al (2003) International physical activity questionnaire: 12-country reliability and validity. Med Sci Sports Exerc 35(8):1381-1395

30. Harris PA, Taylor R, Thielke R, Payne J, Gonzalez N, Conde JG (2009) Research electronic data capture (REDCap)-a metadata-driven methodology and workflow process for providing translational research informatics support. J Biomed Inform 42(2):377-381

31. Perneger TV (1998) What's wrong with Bonferroni adjustments. BMJ 316(7139):1236-1238

32. ABMTRR (2019) Australasian Bone Marrow Transplant Recipient Registry: Annual Data Summary 2018. Australasian Bone Marrow Transplant Recipient Registry, Darlinghurst

33. The Department of Health. Rural, Remote and Metropolitan Areas Classification (RRMA) 2008. Available from: https:// www1.health.gov.au/internet/publications/publishing.nsf/Conte nt/work-res-ruraud-toc $\sim$ work-res-ruraud-lis $\sim$ work-res-ruraud-lise. Accessed 10 Sep 2021

34. SimpleSet. SimpleSet 2009. Available from: https://simpleset.net.

35. Moore J, Merchant Z, Rowlinson K, McEwan K, Evison M, Faulkner $G$ et al (2020) Implementing a system-wide cancer prehabilitation programme: the journey of Greater Manchester's 'Prehab4cancer'. Eur J Surg Oncol

36. Schmitz KH, Campbell AM, Stuiver MM, Pinto BM, Schwartz AL, Morris GS et al (2019) Exercise is medicine in oncology: engaging clinicians to help patients move through cancer. CA Cancer J Clin 69(6):468-484

37. Granger CL, Holland AE, Gordon IR, Denehy L (2015) Minimal important difference of the 6-minute walk distance in lung cancer. Chron Respir Dis 12(2):146-154

38. Gibbons WJ, Fruchter N, Sloan S, Levy RD (2001) Reference values for a multiple repetition 6-minute walk test in healthy adults older than 20 years. J Cardiopulm Rehabil 21(2):87-93

39. Bauer MS, Damschroder L, Hagedorn H, Smith J, Kilbourne AM (2015) An introduction to implementation science for the nonspecialist. BMC Psychol 3:32

40. Santa Mina D, Au D, Auger LE, Alibhai SMH, Matthew AG, Sabiston CM et al (2019) Development, implementation, and effects of a cancer center's exercise-oncology program. Cancer 125(19):3437-3447

41. Poltawski L, Norris M, Dean S (2014) Intervention fidelity: developing an experience-based model for rehabilitation research. J Rehabil Med 46(7):609-615

42. Taylor KL, Weston M, Batterham AM (2015) Evaluating intervention fidelity: an example from a high-intensity interval training study. PLoS ONE 10(4):e0125166

43. Waterland JL, Ismail H, Amin B, Granger CL, Denehy L, Riedel B (2020) Patient acceptance of prehabilitation for major surgery: an exploratory survey. Support Care Cancer 29:779-785

44. Steffens D, Delbaere K, Young J, Solomon M, Denehy L (2020) Evidence on technology-driven preoperative exercise interventions: are we there yet? Br J Anaesth. 125:646-649

Publisher's note Springer Nature remains neutral with regard to jurisdictional claims in published maps and institutional affiliations. 